\title{
Impact of Morphological Status on Long-Term Outcome Among Patients Undergoing Liver Surgery for Intrahepatic Cholangiocarcinoma
}

\author{
Fabio Bagante, MD' ${ }^{1}$, Gaya Spolverato, $\mathrm{MD}^{1}$, Matthew Weiss, $\mathrm{MD}^{2}$, Sorin Alexandrescu, $\mathrm{MD}^{3}$, Hugo P. Marques, \\ $\mathrm{MD}^{4}$, Luca Aldrighetti, $\mathrm{MD}^{5}$, Shishir K. Maithel, $\mathrm{MD}^{6}$, Carlo Pulitano, MD ${ }^{7}$, Todd W. Bauer, $\mathrm{MD}^{8}$, Feng Shen, \\ $\mathrm{MD}^{9}$, George A. Poultsides, MD ${ }^{10}$, Oliver Soubrane, $\mathrm{MD}^{11}$, Guillaume Martel, $\mathrm{MD}^{12}$, B. Groot Koerkamp, $\mathrm{MD}^{13}$, \\ Alfredo Guglielmi, MD ${ }^{1}$, Endo Itaru, $\mathrm{MD}^{14}$, and Timothy M. Pawlik, MD, MPH, PhD ${ }^{15,16}$ \\ ${ }^{1}$ Department of Surgery, University of Verona, Verona, Italy; ${ }^{2}$ Department of Surgery, Johns Hopkins Hospital, Baltimore, \\ MD; ${ }^{3}$ Department of Surgery, Fundeni Clinical Institute, Bucharest, Romania; ${ }^{4}$ Department of Surgery, Curry Cabral \\ Hospital, Lisbon, Portugal; ${ }^{5}$ Department of Surgery, Ospedale San Raffaele, Milan, Italy; ${ }^{6}$ Department of Surgery, Emory \\ University, Atlanta, GA; ${ }^{7}$ Department of Surgery, Royal Prince Alfred Hospital, University of Sydney, Sydney, NSW, \\ Australia; ${ }^{8}$ Department of Surgery, University of Virginia, Charlottesville, VA; ${ }^{9}$ Department of Surgery, Eastern \\ Hepatobiliary Surgery Hospital, Shanghai, China; ${ }^{10}$ Department of Surgery, Stanford University, Stanford, CA; \\ ${ }^{11}$ Department of Hepatobiliopancreatic Surgery and Liver Transplantation, AP-HP, Beaujon Hospital, Clichy, France; \\ ${ }^{12}$ Division of General Surgery, Department of Surgery, University of Ottawa, Ottawa, ON, Canada; ${ }^{13}$ Department of \\ Surgery, Erasmus University Medical Centre, Rotterdam, The Netherlands; ${ }^{14}$ Gastroenterological Surgery Division, \\ Yokohama City University School of Medicine, Yokohama, Japan; ${ }^{15}$ Department of Surgery, The Ohio State University \\ Wexner Medical Center, Columbus, OH; ${ }^{16}$ Department of Surgery, The Urban Meyer III and Shelley Meyer Chair in \\ Cancer Research, The Ohio State University Wexner Medical Center, Columbus, $\mathrm{OH}$
}

\begin{abstract}
Background. The influence of morphological status on the long-term outcome of patients undergoing liver resection for intrahepatic cholangiocarcinoma (ICC) is poorly defined. We sought to study the impact of morphological status on overall survival (OS) of patients undergoing curative-intent resection for ICC.
\end{abstract}

Fabio Bagante and Gaya Spolverato contributed equally to this article.

This paper was presented as an oral presentation at the Society of Surgical Oncology Annual Cancer Symposium, Seattle, WA, USA, 15-18 March 2017.

Electronic supplxlementary material The online version of this article (doi:10.1245/s10434-017-5870-y) contains supplementary material, which is available to authorized users.

(C) Society of Surgical Oncology 2017

First Received: 16 February 2017;

Published Online: 2 May 2017

T. M. Pawlik, MD, MPH, PhD

e-mail: tim.pawlik@osumc.edu
Methods. A total of 1083 patients who underwent liver resection for ICC between 1990 and 2015 were identified. Data on clinicopathological characteristics, operative details, and morphological status were recorded and analyzed. A propensity score-matched analysis was performed to reduce confounding biases.

Results. Among 1083 patients, 941(86.9\%) had a massforming (MF) or intraductal-growth (IG) type, while 142 (13.1\%) had a periductal-infiltrating (PI) or MF with PI components (MF + PI) ICC. Patients with an MF/IG ICC had a 5-year OS of $41.8 \%$ (95\% confidence interval [CI] 37.7-45.9) compared with $25.5 \%$ (95\% CI 17.3-34.4) for patients with a PI/MF + PI $(p<0.001)$. Morphological type was found to be an independent predictor of OS as patients with a PI/MF + PI ICC had a higher hazard of death (hazard ratio [HR] 1.42, 95\% CI 1.11-1.82; $p=0.006)$ compared with patients who had an $\mathrm{MF} / \mathrm{IG}$ ICC. Compared with T1a-T1b-T2 MF/IG tumors, T1aT1b-T2 PI/MF + PI and T3-T4 PI/MF + PI tumors were associated with an increased risk of death (HR 1.47 vs. 3.59). Conversely, patients with T3-T4 MF/IG tumors had a similar risk of death compared with T1a-T1b-T2 MF/IG patients $(p=0.95)$. 
Conclusion. Among patients undergoing curative-intent resection of ICC, morphological status was a predictor of long-term outcome. Patients with PI or MF + PI ICC had an approximately $45 \%$ increased risk of death long-term compared with patients who had an MF or IG ICC.

Intrahepatic cholangiocarcinoma (ICC) is a primary liver tumor that originates from the endothelial cells of segmental or proximal branches of the bile duct. ${ }^{1}$ It accounts for $10-15 \%$ of all primary liver malignancies and its incidence and mortality are increasing worldwide. ${ }^{2}$ Surgical resection remains the cornerstone of potentially curative therapy, however 5-year survival after curative-intent resection remains only $25-30 \% .^{2-11}$ Several studies have identified clinicopathological factors associated with long-term outcomes. $^{2,7,9,12-18}$ Specifically, preoperative cancer antigen (CA) 19-9 levels, tumor number and size, lymph node status, margin status, and vascular invasion have each been associated with an increased risk of death long-term. ${ }^{2,7,9,12-17,19}$

Another less-considered clinicopathological characteristic of ICC involves tumor morphology. The Liver Cancer Study Group of Japan has classified ICC into several categories based on gross appearance: mass-forming (MF) type, periductal-infiltrating (PI) type, and intraductal-growth (IG) type. The MF type is characterized by a defined mass within the liver parenchyma, while the PI subtype extends longitudinally along the bile duct, often with enhancement of the bile duct. In contrast, the IG type grows toward or within the lumen of the bile duct. ${ }^{20}$ While these different ICC morphologies have been defined, few studies have investigated the impact of tumor morphology on long-term prognosis following resection of ICC. ${ }^{21-23}$ Shimada et al. reported that MF + PI ICC had a higher incidence of positive surgical margins, as well as a higher risk of local recurrence. ${ }^{21}$ However, previous studies have been limited due to small patient cohorts derived from single institutions.

Given the lack of data, the objective of the current study was to define the impact of ICC morphologic status relative to long-term outcomes following liver resection for ICC using a large multi-institutional international cohort. In addition, we sought to define the relationship of tumor morphology and the American Joint Committee on Cancer (AJCC) 8th edition $\mathrm{T}$ categories.

\section{INTRODUCTION}

\section{PATIENTS AND METHODS}

\section{Study Population and Data Collection}

Patients who underwent hepatic resection for histologically confirmed ICC at one of 14 major hepatobiliary centers between 1990 and 2015 were identified. The 14 medical centers included Johns Hopkins Hospital, Baltimore, MD, USA; Stanford University, Stanford, CA, USA; University of Virginia, Charlottesville, VA, USA; Emory University, Atlanta, GA, USA; Fundeni Clinical Institute of Digestive Disease, Bucharest, Romania; Curry Cabral Hospital, Lisbon, Portugal; Ospedale San Raffaele, Milan, Italy; Royal Prince Alfred Hospital, University of Sydney, Sydney, NSW, Australia; Eastern Hepatobiliary Surgery Hospital, Shanghai, China; Beaujon Hospital, Clichy, France; University of Ottawa, Ottawa, ON, Canada; Erasmus University Medical Centre, Rotterdam, The Netherlands; Yokohama City University School of Medicine, Yokohama, Japan; and University of Verona, School of Medicine, Verona, Italy. The Institutional Review Board of the participating institutions approved the study. Only patients who underwent curative-intent liver resection for nonmetastatic tumors were included. Patients who underwent a noncurative resection (R2), as well as patients who received only ablation or intra-arterial therapy, were excluded.

Standard patient demographic and clinicopathological characteristics were collected, including age, sex, American Society of Anesthesiologists (ASA) class, presence of cirrhosis in the underlying liver, and serum level of carcinoembryonic antigen (CEA) and CA19-9. ICC-specific characteristics, including tumor location, tumor burden, invasion of adjacent organs, liver capsule involvement, margin status, tumor grade, major vascular/lymphovascular/perineural invasion, and nodal status were collected. For the purposes of analyses, patients were grouped as $\mathrm{MF} /$ IG type versus PI/MF with PI components (MF + PI) ICC. For all cases, the imaging and pathological data were reviewed to determine the macroscopic morphologic subtype. In addition, since early 2000 , ICC morphological type has been included in the pathological report as a standard data field regarding tumor characteristics at the 14 participating centers. Treatment-related data such as type and extent of hepatic resection, lymphadenectomy, and receipt of neoadjuvant chemotherapy were also recorded. Tumor stage was categorized according to the 8th edition of the AJCC. ${ }^{24}$ The presence of multifocal ICC, invasion of adjacent organs, liver capsule involvement, margin status, tumor grade, vascular/lymphovascular/perineural invasion, nodal status, morphological type, and AJCC stage were determined based on final pathological reports.

\section{Statistical Analysis}

Discrete variables were described as medians with interquartile range (IQR) and categorical variables were recorded as totals and frequencies. Univariable comparisons were assessed using the Chi square test or Fisher's 
exact test as appropriate. Univariable and multivariable analyses were performed using Cox proportional hazard regression models to assess possible associations among covariates. Variables significant on univariable analysis $(p<0.05)$ were entered into the multivariable model and backward selection was used to eliminate nonsignificant variables at $p<0.10$. Furthermore, to account for any potential residual confounders in the effect of morphologic subtype classification on survival, propensity scores were estimated using a logistic regression model, with morphological type specified as MF/IG versus $\mathrm{PI} / \mathrm{MF}+\mathrm{PI}$. Age, ASA score, underlying liver disease, neoadjuvant chemotherapy, type of surgery, margin status, liver capsule involvement, invasion of adjacent organs, tumor size and number, tumor differentiation, major vascular invasion, lymphovascular invasion, perineural invasion, lymph node status, and AJCC 8th edition T-staging system were included as independent variables in the logistic regression model. For matching, a caliper width of 0.1 times the standard deviation of the propensity score was used. The degrees of covariate imbalance in unmatched and matched samples were measured using the standardized (mean and proportion) differences as proposed by Austin et al. ${ }^{25} \mathrm{~A}$ $p$ value $<0.05$ (two-tailed) was considered statistically significant. All analyses were performed using STATA version 12.0 (StataCorp LP, College Station, TX, USA) or $\mathrm{R}$ software for statistical computing, v. 3.0.2 34, with the additional packages, survival, Hmisc and Matching.

\section{RESULTS}

\section{Baseline Characteristics of the Study Group Stratified by the Intrahepatic Cholangiocarcinoma Morphological Types}

Among 1083 patients who underwent liver resection for ICC, $911(84.1 \%)$ patients had a MF type ICC, $30(2.8 \%)$ had an IG type, $54(5.0 \%)$ had a PI type, and $88(8.1 \%)$ patients had an MF + PI type ICC. Accordingly, 941 (86.9\%) patients were included in the MF/IG group, while $142(13.1 \%)$ were included in the PI/MF + PI group (Table 1). While most clinical characteristics were comparable in the two groups, patients with MF/IG ICC were younger than patients with PI/MF + PI ICC tumors (MF/ IG 59 years vs. PI/MF + PI 63 years; $p=0.008$ ) and had a lower ASA score (ASA score >2, MF/IG: $53.5 \%$ vs. PI/ MF + PI: $41.8 \% ; p=0.008)$. While preoperative tumor markers such as CEA and CA19-9 were similar (both $p$ value $>0.10$ ), patients with $\mathrm{PI} / \mathrm{MF}+\mathrm{PI}$ ICC tumors $(27.9 \% ; n=24)$ were more likely to have received neoadjuvant chemotherapy compared with $\mathrm{MF} / \mathrm{IG}$ patients $(6.8 \% ; n=53) \quad[p<0.001]$. While the overwhelming majority of patients with PI/MF + PI tumors underwent a major hepatectomy $(n=134,94.4 \%)$, only approximately one-half of patients with MF/IG tumors $(n=512,54.4 \%)$ had a major resection $(p<0.001)$. Lymphadenectomy was also more often performed among patients with PI/ $\mathrm{MF}+\mathrm{PI} \quad(72.5 \%)$ versus $\mathrm{MF} / \mathrm{IG} \quad(40.7 \%)$ tumors $(p<0.001)$.

On final pathology, tumor size and number were comparable among patients with $\mathrm{PI} / \mathrm{MF}+\mathrm{PI}$ versus $\mathrm{MF} / \mathrm{IG}$ tumors, as was the incidence of poor/undifferentiated ICC tumors (Table 1). In contrast, compared with MF/IG patients, PI/MF + PI tumors were more likely to be associated with major vascular invasion (PI/MF + PI 26.8\% vs. MF/IG 9.5\%), as well as lymphovascular (PI/MF + PI $46.1 \%$ vs. $\mathrm{MF} / \mathrm{IG} 28.8 \%$ ) and perineural invasion (PI/ $\mathrm{MF}+\mathrm{PI} 37.7 \%$ vs. MF/IG 17.9\%) [all $p<0.001$ ]. In addition, patients with $\mathrm{PI} / \mathrm{MF}+\mathrm{PI}$ tumors were more likely to have undergone a margin positive resection (PI/ $\mathrm{MF}+\mathrm{PI} 23.4 \%$ vs. MF/IG $10.8 \% ; p<0.001)$. PI/ $\mathrm{MF}+$ PI patients also had a higher incidence of harboring metastatic lymph node disease (PI/MF + PI 59.2\% vs. MF/ IG $34.7 \% ; p<0.001)$.

Using the 8th edition of the AJCC staging system, PI/ $\mathrm{MF}+\mathrm{PI}$ patients had more advanced T categories (T2/T3/ T4, PI/MF + PI 77.5\% vs. MF/IG 51.4\%; $p<0.001)$, as well as $\mathrm{N}$ status (N1, PI/MF + PI 77.2\% vs. MF/IG:58.5\%; $p=0.003$ ). Accordingly, $95.0 \%$ of PI/MF + PI patients were staged as II/IIIa/IIIb versus $86.0 \%$ of MF/IG patients $(p=0.017)$.

\section{Univariable and Multivariable Survival Analyses}

Within a median follow-up of 1.7 years (IQR 0.91-3.46), 537 (49.6\%) patients died; 3- and 5-year overall survival (OS) was $51.9 \%$ (95\% confidence interval [CI] 48.6-55.3) and 39.5\% (95\% CI 35.7-43.3), respectively. Several variables were associated with OS on univariable analysis, including tumor markers (CEA and CA 19-9), type of surgery, margin status, invasion of adjacent organs, tumor size and number, tumor differentiation, major vascular invasion, lymphovascular invasion, perineural invasion, nodal status, and AJCC 8th edition staging (Tables 2, 3). In addition, patients with an MF/IG ICC had a 5-year OS of $41.8 \%$ (95\% CI 37.7-45.9), which was markedly better than the $25.5 \%$ (95\% CI $17.3-34.4$ ) among patients with a PI/MF + PI tumor $(p<0.001)$ [Fig. 1].

On multivariable analysis, tumor size $(>5 \mathrm{~cm}$; hazard ratio [HR] $1.75,95 \%$ CI 1.44-2.13), invasion of adjacent organs (HR 1.75, 95\% CI 1.28-2.38), and metastatic nodal status (HR 2.42, 95\% CI 1.84-3.18) [all $p<0.001$ ] remained strongly associated with OS (Table 4). In addition, positive margin status (HR 1.38, 95\% CI 1.06-1.79), 
TABLE 1 Baseline characteristics $(n=1083)$

\begin{tabular}{|c|c|c|c|c|}
\hline Variables & ICC & $\mathrm{MF} / \mathrm{IG}$ & $\mathrm{PI} / \mathrm{MF}+\mathrm{PI}$ & $p$ Value \\
\hline No. of patients & 1083 & 941 & 142 & \\
\hline Sex & & & & 0.88 \\
\hline Female & $466(43.1)$ & $404(42.9)$ & $62(43.7)$ & \\
\hline Male & $617(56.9)$ & $537(57.1)$ & $80(56.3)$ & \\
\hline Age, years [median (IQR)] & $60(51-69)$ & $59(51-68)$ & $63(56-71)$ & 0.008 \\
\hline ASA score & & & & 0.008 \\
\hline $1-2$ & $614(56.7)$ & $548(58.2)$ & $66(46.5)$ & \\
\hline $3-4$ & $469(43.3)$ & $393(41.8)$ & $76(53.5)$ & \\
\hline Underlying liver disease & & & & $<0.001$ \\
\hline Cirrhosis & $117(10.8)$ & $112(11.9)$ & $5(3.5)$ & \\
\hline Chronic HBV infection & $204(18.8)$ & $196(20.8)$ & $8(5.6)$ & \\
\hline Chronic HCV infection & $31(2.9)$ & $21(2.2)$ & $10(7.1)$ & \\
\hline None & $731(67.9)$ & $612(65.1)$ & $119(83.8)$ & \\
\hline Neoadjuvant chemotherapy & & & & $<0.001$ \\
\hline No & $783(91.1)$ & $721(93.2)$ & $62(72.1)$ & \\
\hline Yes & $77(8.9)$ & $53(6.8)$ & $24(27.9)$ & \\
\hline NA & 223 & 167 & 56 & \\
\hline CA19-9, U/mL [median (IQR)] & $47(17-190)$ & $45(16-174)$ & $94(26-494)$ & 0.12 \\
\hline CEA, ng/mL [median (IQR)] & $2.4(1.4-4.3)$ & $2.4(1.4-4.2)$ & $2.6(1.4-5.5)$ & 0.72 \\
\hline Type of surgery & & & & $<0.001$ \\
\hline Wedge resection & $169(15.6)$ & $169(18.0)$ & $0(0)$ & \\
\hline Minor hepatectomy & $268(24.7)$ & $260(27.6)$ & $8(5.6)$ & \\
\hline Major hepatectomy & $646(59.7)$ & $512(54.4)$ & $134(94.4)$ & \\
\hline Margins & & & & $<0.001$ \\
\hline Negative & 945 (87.6) & $837(89.2)$ & $108(76.6)$ & \\
\hline Positive & $134(12.4)$ & $101(10.8)$ & $33(23.4)$ & \\
\hline NA & 4 & 3 & 1 & \\
\hline Liver capsule involvement & & & & 0.25 \\
\hline No & $877(80.9)$ & $767(81.5)$ & $110(77.5)$ & \\
\hline Yes & $206(19.1)$ & $174(18.5)$ & $32(22.5)$ & \\
\hline Invasion of adjacent organs & & & & $<0.001$ \\
\hline No & $1017(93.9)$ & $901(95.7)$ & $116(81.7)$ & \\
\hline Yes & $66(6.1)$ & $40(4.3)$ & $26(18.3)$ & \\
\hline Tumor size, $\mathrm{cm}$ & & & & 0.06 \\
\hline$\leq 5$ & $434(40.1)$ & $367(39.0)$ & $67(47.2)$ & \\
\hline$>5$ & $649(59.9)$ & $574(61.0)$ & $75(52.8)$ & \\
\hline Lesion & & & & 0.12 \\
\hline Unifocal & $890(82.2)$ & $780(82.9)$ & $110(77.5)$ & \\
\hline Multifocal & $193(17.8)$ & $161(17.1)$ & $32(22.5)$ & \\
\hline Grade & & & & 0.22 \\
\hline Well-moderate & $845(83.0)$ & $742(83.6)$ & $103(79.2)$ & \\
\hline Poor-undifferentiated & $173(17.0)$ & $146(16.4)$ & $27(20.8)$ & \\
\hline NA & 65 & 53 & 12 & \\
\hline Major vascular invasion & & & & $<0.001$ \\
\hline Not present & $956(88.3)$ & $852(90.5)$ & $104(73.2)$ & \\
\hline Present & $127(11.7)$ & $89(9.5)$ & $38(26.8)$ & \\
\hline Lymphovascular invasion & & & & $<0.001$ \\
\hline Not present & $736(68.9)$ & $660(71.2)$ & $76(53.9)$ & \\
\hline
\end{tabular}


TABLE 1 continued

\begin{tabular}{|c|c|c|c|c|}
\hline Variables & $\mathrm{ICC}$ & $\mathrm{MF} / \mathrm{IG}$ & $\mathrm{PI} / \mathrm{MF}+\mathrm{PI}$ & $p$ Value \\
\hline Present & $332(31.1)$ & $267(28.8)$ & $65(46.1)$ & \\
\hline NA & 15 & 14 & 1 & \\
\hline Perineural invasion & & & & $<0.001$ \\
\hline Not present & $787(79.5)$ & $706(82.1)$ & $81(62.3)$ & \\
\hline Present & $203(20.5)$ & $154(17.9)$ & $49(37.7)$ & \\
\hline NA & 96 & 81 & 12 & \\
\hline Lymphadenectomy & & & & $<0.001$ \\
\hline Not performed & $597(55.1)$ & $558(59.3)$ & $39(27.5)$ & \\
\hline Performed & $486(44.9)$ & $383(40.7)$ & $103(72.5)$ & \\
\hline Pathological nodal status ${ }^{\mathrm{a}}$ & & & & $<0.001$ \\
\hline Negative & $292(60.1)$ & $250(65.3)$ & $42(40.8)$ & \\
\hline Positive & $194(39.9)$ & $133(34.7)$ & $61(59.2)$ & \\
\hline AJCC 8th edition $\mathrm{N}$ categories ${ }^{\mathrm{b}}$ & & & & 0.003 \\
\hline No & $114(36.7)$ & $96(41.5)$ & $18(22.7)$ & \\
\hline N1 & $194(63.3)$ & $133(58.5)$ & $61(77.2)$ & \\
\hline AJCC 8th edition $\mathrm{T}$ categories & & & & $<0.001$ \\
\hline T1a & 238 (21.9) & $223(23.7)$ & $15(10.6)$ & \\
\hline $\mathrm{T} 1 \mathrm{~b}$ & $252(23.3)$ & $235(24.9)$ & $17(11.9)$ & \\
\hline $\mathrm{T} 2$ & $363(33.5)$ & $291(30.9)$ & $72(50.7)$ & \\
\hline $\mathrm{T} 3$ & $164(15.1)$ & $152(16.2)$ & $12(8.5)$ & \\
\hline $\mathrm{T} 4$ & $66(6.1)$ & $40(4.3)$ & $26(18.3)$ & \\
\hline AJCC 8th edition stages ${ }^{\mathrm{b}}$ & & & & 0.017 \\
\hline Ia & $15(4.9)$ & $15(6.6)$ & $0(0)$ & \\
\hline $\mathrm{Ib}$ & $21(6.8)$ & $17(7.4)$ & $4(5.0)$ & \\
\hline II & $47(15.3)$ & $37(16.2)$ & $10(12.7)$ & \\
\hline IIIa & $26(8.4)$ & $23(10.0)$ & $3(3.8)$ & \\
\hline IIIb & $199(64.6)$ & $137(59.8)$ & $62(78.5)$ & \\
\hline
\end{tabular}

Data are expressed as $n(\%)$ unless otherwise specified

$N A$ not available, $I C C$ intrahepatic cholangiocarcinoma, $M F$ mass forming, $I G$ intraductal growth, $P I$ periductal infiltrating, $I Q R$ interquartile range, $A S A$ American Society of Anesthesiologists, $H B V$ hepatitis B virus, $H C V$ hepatitis $\mathrm{C}$ virus, $C A$ cancer antigen, $C E A$ carcinoembryonic antigen, AJCC American Joint Committee on Cancer

${ }^{\text {a }}$ Patients who underwent lymphadenectomy $(N=486)$

${ }^{\mathrm{b}}$ Patients who had at least six lymph nodes harvested $(N=308)$

multifocal ICC (HR 1.38, 95\% CI 1.11-1.72), poor/undifferentiated tumor grade (HR 1.60, 95\% CI 1.28-2.01), and perineural invasion (HR 1.28, 95\% CI 1.01-1.62) [all $p<0.05$ ] were also associated with a poor prognosis. Of note, after controlling for these competing risk factors, morphological subtype remained associated with OS as patients with a PI/MF + PI ICC had an approximately $40 \%$ higher hazard of death compared with patients who had MF/IG ICC (HR 1.42, 95\% CI 1.11-1.82) [ $p=0.006]$.

\section{Prognosis of Patients and Morphological Types}

Given the baseline differences between the MF/IG and $\mathrm{PI} / \mathrm{MF}+\mathrm{PI}$ groups, a propensity score-matching analysis was then performed to minimize potential confounding.
Patients were matched based on age, ASA score, underlying liver disease, neoadjuvant chemotherapy, type of surgery, margin status, invasion of adjacent organs, tumor size and number, tumor differentiation, vascular invasion, lymphovascular invasion, perineural invasion, lymph node status, and AJCC 8th edition T categories.

After the propensity score matching, 95 patients in the $\mathrm{PI} / \mathrm{MF}+\mathrm{PI}$ group and 95 patients in the MF/IG group had comparable characteristics and were subsequently analyzed (electronic supplementary Table S1) [all $p>0.1$ ]. In the propensity score-matched analysis, individuals with $\mathrm{MF} / \mathrm{IG}$ ICC still had a more favorable prognosis compared with patients who had PI/MF + PI tumors (5-year OS: MF/IG $35.7 \%, 95 \%$ CI $24.0-47.6$ vs. PI/MF + PI $26.2 \%, 95 \%$ CI 16.4-37.1) [ $p=0.03]$. 
TABLE 2 Univariate survival analysis $(n=1083)$

\begin{tabular}{|c|c|c|c|c|}
\hline Variables & $N=1083[n(\%)]$ & 5 -year OS (\%) & $95 \% \mathrm{CI}$ & $p$ Value \\
\hline \multicolumn{5}{|l|}{ Sex } \\
\hline Female & $66(43.1)$ & 37.7 & $32.7-42.7$ & 0.28 \\
\hline Male & $617(56.9)$ & 41.9 & $36.1-47.6$ & \\
\hline Age, years & & & & 0.63 \\
\hline$\leq 65$ & $675(62.3)$ & 39.4 & $34.7-44.0$ & \\
\hline$>65$ & $408(37.7)$ & 39.5 & $33.1-45.8$ & \\
\hline ASA score & & & & 0.09 \\
\hline $1-2$ & $614(56.7)$ & 40.9 & $35.6-46.0$ & \\
\hline $3-4$ & $469(43.3)$ & 37.4 & $31.9-42.9$ & \\
\hline Underlying liver disease & & & & $<0.001$ \\
\hline Cirrhosis & $117(10.8)$ & $112(11.9 \%)$ & $5(3.5 \%)$ & \\
\hline Chronic HBV infection & $204(18.8)$ & $196(20.8 \%)$ & $8(5.6 \%)$ & \\
\hline Chronic HCV infection & $31(2.9)$ & $21(2.2 \%)$ & $10(7.1 \%)$ & \\
\hline None & $731(67.9)$ & $612(65.1 \%)$ & $119(83.8 \%)$ & \\
\hline Morphological types & & & & $<0.001$ \\
\hline MF/IG & $941(86.9)$ & 41.8 & $37.7-45.9$ & \\
\hline $\mathrm{PI} / \mathrm{MF}+\mathrm{PI}$ & $142(13.1)$ & 25.5 & $17.3-34.4$ & \\
\hline Neoadjuvant chemotherapy & & & & 0.69 \\
\hline No & $783(91.1)$ & 44.0 & $39.3-48.6$ & \\
\hline Yes & $77(8.9)$ & 42.5 & $26.8-57.3$ & \\
\hline CA19-9, U/mL & & & & $<0.001$ \\
\hline$\leq 50$ & $400(51.4)$ & 50.7 & $44.2-56.9$ & \\
\hline$>50$ & $379(48.5)$ & 31.5 & $25.6-37.5$ & \\
\hline CEA, ng/mL & & & & $<0.001$ \\
\hline$\leq 10$ & $592(89.9)$ & 42.9 & $37.5-48.2$ & \\
\hline$>10$ & $66(10.1)$ & 4.2 & $0.4-16.4$ & \\
\hline Type of surgery & & & & 0.011 \\
\hline Wedge resection & 169 (15.6) & 40.2 & $29.4-50.7$ & \\
\hline Minor hepatectomy & $268(24.7)$ & 49.1 & $40.8-56.8$ & \\
\hline Major hepatectomy & $646(59.7)$ & 37.8 & $32.8-42.8$ & \\
\hline Margins & & & & $<0.001$ \\
\hline Negative & 945 (87.6) & 40.9 & $36.9-44.9$ & \\
\hline Positive & $134(12.4)$ & 29.6 & $20.1-39.6$ & \\
\hline \multicolumn{5}{|l|}{ Liver capsule involvement } \\
\hline No & $877(80.9)$ & 39.7 & $35.4-43.9$ & \\
\hline Yes & $206(19.1)$ & 38.5 & $30.7-46.3$ & 0.47 \\
\hline Invasion of adjacent organs & & & & $<0.001$ \\
\hline No & 1017 (93.9) & 43.3 & $39.1-47.5$ & \\
\hline Yes & $66(6.1)$ & 14.7 & $6.4-26.5$ & \\
\hline Tumor size, $\mathrm{cm}$ & & & & $<0.001$ \\
\hline$\leq 5$ & $434(40.1)$ & 51.9 & $45.9-57.7$ & \\
\hline$>5$ & $649(59.9)$ & 30.7 & $26.1-35.5$ & \\
\hline Lesion & & & & $<0.001$ \\
\hline Unifocal & $890(82.2)$ & 43.3 & $39.0-47.5$ & \\
\hline Multifocal & 193 (17.8) & 22.9 & $16.0-30.6$ & \\
\hline Grade & & & & $<0.001$ \\
\hline Well-moderate & $845(83.0)$ & 42.9 & $38.6-47.2$ & \\
\hline Poor-undifferentiated & $173(17.0)$ & 22.3 & $14.9-30.7$ & \\
\hline
\end{tabular}


TABLE 2 continued

\begin{tabular}{|c|c|c|c|c|}
\hline Variables & $N=1083[n(\%)]$ & 5-year OS (\%) & $95 \% \mathrm{CI}$ & $p$ Value \\
\hline Major vascular invasion & & & & $<0.001$ \\
\hline Not present & $956(88.3)$ & 41.6 & $37.5-45.6$ & \\
\hline Present & $127(11.7)$ & 26.4 & $17.4-36.2$ & \\
\hline Lymphovascular invasion & & & & 0.004 \\
\hline Not present & $736(68.9)$ & 43.0 & $38.5-47.4$ & \\
\hline Present & $332(31.1)$ & 31.6 & $26.7-38.7$ & \\
\hline Perineural invasion & & & & 0.001 \\
\hline Not present & $787(79.5)$ & 42.7 & $38.4-46.9$ & \\
\hline Present & $203(20.5)$ & 22.1 & $13.7-31.8$ & \\
\hline Lymphadenectomy & & & & 0.07 \\
\hline Not performed & $597(55.1)$ & 43.9 & $38.9-48.8$ & \\
\hline Performed & $486(44.9)$ & 33.4 & $27.7-39.1$ & \\
\hline Pathological nodal status ${ }^{\mathrm{a}}$ & & & & $<0.001$ \\
\hline Negative & $292(60.1)$ & 44.3 & $36.6-51.7$ & \\
\hline Positive & $194(39.9)$ & 15.7 & $8.9-24.1$ & \\
\hline \multicolumn{5}{|l|}{ AJCC 8 th edition $\mathrm{N}$ categories } \\
\hline No & $114(36.7)$ & 54.5 & $40.9-66.2$ & $<0.001$ \\
\hline N1 & $194(63.3)$ & 15.6 & $8.9-23.9$ & \\
\hline AJCC 8th edition $\mathrm{T}$ categories & & & & $<0.001$ \\
\hline $\mathrm{T} 1 \mathrm{a}$ & $238(21.9)$ & 60.9 & $52.7-68.2$ & \\
\hline $\mathrm{T} 1 \mathrm{~b}$ & $252(23.3)$ & 33.9 & $26.0-41.8$ & \\
\hline $\mathrm{T} 2$ & $363(33.5)$ & 29.9 & $23.6-36.5$ & \\
\hline $\mathrm{T} 3$ & $164(15.1)$ & 45.8 & $36.6-54.6$ & \\
\hline $\mathrm{T} 4$ & $66(6.1)$ & 14.7 & $6.4-26.5$ & \\
\hline AJCC 8th edition stages ${ }^{\mathrm{b}}$ & & & & $<0.001$ \\
\hline Ia & $15(4.9)$ & 90.9 & $50.8-98.7$ & \\
\hline $\mathrm{Ib}$ & $21(6.8)$ & 51.9 & $23.4-74.3$ & \\
\hline II & $47(15.3)$ & 48.0 & $27.7-65.7$ & \\
\hline IIIa & $26(8.4)$ & 42.5 & $13.4-69.4$ & \\
\hline IIIb & $199(64.6)$ & 16.6 & $9.8-25.1$ & \\
\hline
\end{tabular}

$N A$ not available, $O S$ overall survival, $C I$ confidence interval, $M F$ mass forming, $I G$ intraductal growth, $P I$ periductal infiltrating, $A S A$ American Society of Anesthesiologists, $H B V$ hepatitis B virus, $H C V$ hepatitis $\mathrm{C}$ virus, $C A$ cancer antigen, $C E A$ carcinoembryonic antigen, $A J C C$ American Joint Committee on Cancer

${ }^{\text {a }}$ Patients who underwent lymphadenectomy $(N=486)$

b Patients who had at least six lymph nodes harvested $(N=308)$

\section{American Joint Committee on Cancer 8th edition T categories and Morphological Types}

Patients were then stratified according to the AJCC 8th edition $\mathrm{T}$ categories, as well as morphologic subtypes (Tables 5, 6). A total of $749(69.2 \%)$ patients were in the MF/IG group and in T1a-T1b-T2 stages compared with only $104(9.6 \%)$ patients in the PI/MF + PI group and T1aT1b-T2 stages. Furthermore, 192 (17.7\%) patients were in the MF/IG group and in T3-T4 stages, while 38 (3.5\%) patients were in the PI/MF + PI group and in T3-T4 stages. Of note, even among the T1a-T1b-T2 categories, patients with MF/IG morphology had a better 5-year OS of $41.6 \%$ (95\% CI 36.9-46.3) versus $32.0 \%$ (95\% CI 21.6-42.9) for patients with a PI/MF + PI ICC $(p<0.001)$. Similarly, among patients categorized as T3-T4, 5-year OS following resection of an MF/IG ICC tumor was $42.5 \%$ (95\% CI 34.1-50.7) compared with only $5.6 \%$ (95\% CI 0.5-20.6; $p<0.001$ ) for PI/MF + PI ICC tumors (Fig. 2). Moreover, compared with T1a-T1b-T2 MF/IG tumors, T1a-T1b-T2 $\mathrm{PI} / \mathrm{MF}+\mathrm{PI}$ (HR 1.47, 95\% CI 1.11-1.94) and T3-T4 PI/ $\mathrm{MF}+\mathrm{PI}$ (HR 3.59, 95\% CI 2.46-5.23) lesions were associated with an increased risk of death. In contrast, patients with a T3-T4 MF/IG tumor had a comparable risk of death 
TABLE 3 Comparison between morphological types-Kaplan-Meier analysis

\begin{tabular}{llll}
\hline & $N=1083[n(\%)]$ & 5 -year OS $(\%)$ & $95 \%$ CI \\
\hline Morphological types & & & $<0.001$ \\
MF/IG & $941(86.9)$ & 41.8 & $37.7-45.9$ \\
PI/MF + PI & $142(13.1)$ & 25.5 & $17.3-34.4$ \\
Morphological types_after PS & $N=190$ & & \\
MF/IG & $95(50.0)$ & 35.7 & $24.0-47.6$ \\
PI/MF + PI & $95(50.0)$ & 26.2 & $16.4-37.1$ \\
\hline
\end{tabular}

$O S$ overall survival, $C I$ confidence interval, $M F$ mass forming, $I G$ intraductal growth, $P I$ periductal infiltrating

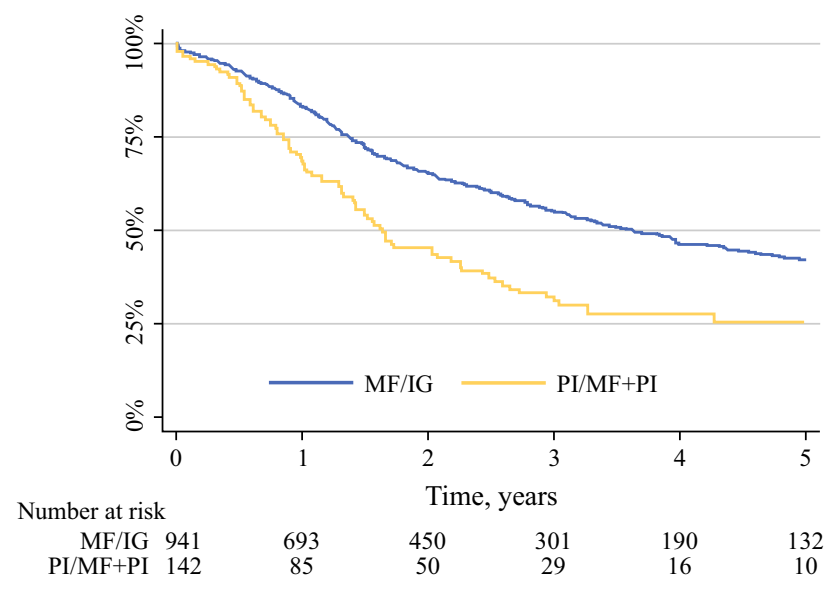

FIG. 1 Kaplan-Meier overall survival curves stratified by morphological type classification. $M F$ mass forming, $I G$ intraductal growth, PI periductal infiltrating

versus patients who had a T1a-T1b-T2 MF/IG lesion (HR $1.01,95 \%$ CI $0.79-1.28)[p=0.95]$.

\section{DISCUSSION}

Similar to other solid malignancies, there has been significant interest in identifying clinicopathological factors associated with survival among patients undergoing curative-intent surgery for ICC. To this end, several groups have proposed various staging and prognostic schemes to stratify ICC patients with regard to prognosis. ${ }^{2,26}$ For example, Hyder et al. reported a nomogram that included six factors, including age, tumor size, number of lesions, nodal status, vascular invasion, and the presence of cirrhosis. ${ }^{26}$ While the nomogram performed reasonably well, the overall accuracy was only moderate, with a C-statistic of 0.706. Such data suggest that other factors may be important in stratifying patient prognosis following resection of ICC. One such possible factor may be tumor morphology, which was first classified by the Liver Cancer Study of Japan. ${ }^{20}$ To date, most studies on ICC have failed to consider, or even report, the morphologic subtype of ICC tumors included in the analytic cohort. ${ }^{2,7,9,12-17}$ The current study is important because we were able to examine the impact of morphologic ICC subtype in a large, multicenter cohort of over 1000 patients undergoing surgery for ICC at one of 14 major hepatobiliary centers in the US, Europe, Australia, and Asia. Specifically, we noted that patients with an MF/IG ICC had a much more favorable prognosis compared with patients who had PI/MF + PI tumors. In particular, PI/MF + PI tumors were associated with many more aggressive features than $\mathrm{MF} / \mathrm{IG}$ lesions. Interestingly, even after controlling for these competing risk factors, morphologic subtype remained associated with long-term survival. Moreover, when patients were stratified by the AJCC 8th edition staging, ICC tumor morphology was still associated with prognosis within T-category subgroups (Tables 5, 6).

Similar to previous reports, clinical variables associated with survival included lymph node status, tumor size and number, positive margin status, tumor grade, and lymphovascular and perineural invasion. ${ }^{6,27}$ More importantly, ICC morphologic type was also strongly associated with OS. Specifically, patients with an MF/IG ICC had a 5-year OS of $41.8 \%$ versus $25.5 \%$ for patients with a PI/MF + PI tumor $(p<0.001)$. Of note, after propensity score matching to control for potential confounding, MF/IG patients still were noted to have a better OS compared with patients who had PI/MF + PI tumors $(35.7 \%$ vs. $26.2 \%$, respectively). These data were consistent with Shimada et al., who reported that patients with MF ICC tumors had a more favorable prognosis versus the MF + PI subtype. ${ }^{21}$ Furthermore, in a separate small study on 52 patients undergoing curative-intent surgery for ICC, Guglielmi et al. reported that patients with MF tumors (50 months) had a markedly longer median survival compared with either the MF/PI subtype (19 months) or the pure PI subtype (15 months).$^{28}$ In addition, in the current study, the MF + PI ICC subtype was associated with more aggressive tumor characteristics, which was consistent with the study by Shimada et al., which reported MF + PI ICC 
TABLE 4 Multivariable survival analysis-Cox's model

\begin{tabular}{|c|c|c|c|}
\hline Variables & HR & $95 \% \mathrm{CI}$ & $p$ Value \\
\hline Invasion of adjacent organs & & & $<0.001$ \\
\hline No & - & - & \\
\hline Yes & 1.75 & $1.28-2.38$ & \\
\hline Margins & & & 0.015 \\
\hline Negative & - & - & \\
\hline Positive & 1.38 & $1.06-1.79$ & \\
\hline Tumor size, $\mathrm{cm}$ & & & $<0.001$ \\
\hline$\leq 5$ & - & - & \\
\hline$>5$ & 1.75 & $1.44-2.13$ & \\
\hline Lesion & & & 0.003 \\
\hline Unifocal & - & - & \\
\hline Multifocal & 1.38 & $1.11-1.72$ & \\
\hline Grade & & & $<0.001$ \\
\hline Well-moderate & - & - & \\
\hline Poor-undifferentiated & 1.60 & $1.28-2.01$ & \\
\hline Perineural invasion & & & 0.043 \\
\hline Not present & - & - & \\
\hline Present & 1.28 & $1.01-1.62$ & \\
\hline Pathological nodal status ${ }^{\mathrm{a}}$ & & & $<0.001$ \\
\hline Negative & - & - & \\
\hline Positive & 2.42 & $1.84-3.18$ & \\
\hline Not harvested & 1.59 & $1.27-2.02$ & \\
\hline Morphological types & & & 0.006 \\
\hline MF/IG & - & - & \\
\hline $\mathrm{PI} / \mathrm{MF}+\mathrm{PI}$ & 1.42 & $1.11-1.82$ & \\
\hline
\end{tabular}

$H R$ hazard ratio, $C I$ confidence interval, $M F$ mass forming, $I G$ intraductal growth, $P I$ periductal infiltrating

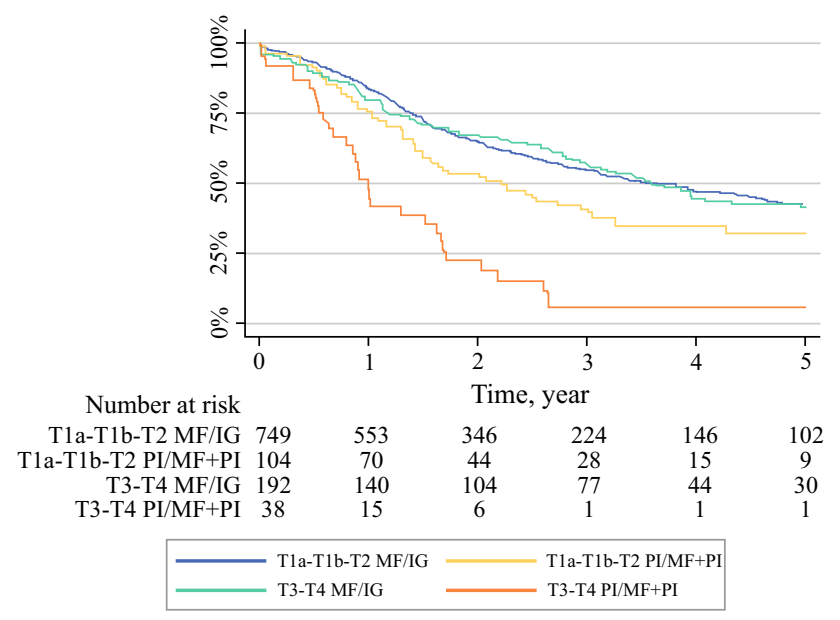

FIG. 2 Kaplan-Meier overall survival curves stratified by the AJCC 8th edition T-category staging and morphological type classification. AJCC American Joint Committee on Cancer, $M F$ mass forming, $I G$ intraductal growth, $P I$ periductal infiltrating

macroscopic tumor types to be associated with increased risk of jaundice, bile duct invasion, portal vein invasion, lymph node metastasis, and R1 margins. ${ }^{21}$
To further evaluate the prognostic effect of gross tumor morphology, prognosis was stratified by AJCC 8th edition $\mathrm{T}$ categories by morphological subtype. Among T1a-T1bT2 patients (i.e. solitary tumor measuring $\leq 5 \mathrm{~cm}$ [T1a]; solitary tumor $>5 \mathrm{~cm}$ [T1b]; solitary tumor with intrahepatic vascular invasion or multiple tumors, with or without vascular invasion [T2]), patients with MF/IG ICC had a 5-year OS of 41.6 versus $32.0 \%$ for patients with PI/ $\mathrm{MF}+\mathrm{PI}$ tumors $(p<0.001)$. A similar difference in survival based on ICC morphologic subtype was noted among patients with T3-T4 tumors. Interestingly, T3-T4 MF/IG patients had a similar risk of death as patients with T1aT1b-T2 MF/IG tumors $(p=0.95)$. Similarly, in a recent paper from our group comparing the AJCC 7th versus 8th edition staging systems for ICC, 8th edition T3 patients paradoxically had a better 5-year OS than either T1 or T2 patients. $^{29}$ These data, in conjunction with data from the current study, suggest that factors other than those currently included in the AJCC staging manual are needed to improve prognostication of survival among patients with ICC. 
TABLE 5 Multivariable survival analysis-Cox's model with AJCC 8th edition T categories

\begin{tabular}{lllr}
\hline Variables & HR & $95 \%$ CI & $p$ Value \\
\hline AJCC 8th edition T categories & & & \\
T1a & - & - & $<-$ \\
T1b & 2.01 & $1.49-2.70$ & $<0.001$ \\
T2 & 2.27 & $1.71-3.00$ & 0.001 \\
T3 & 1.60 & $2.71-5.75$ & $<0.001$ \\
T4 & 3.94 & & 0.003 \\
Morphological types & & & \\
MF/IG & - & - & $1.14-1.84$ \\
PI/MF + PI & 1.45 & & \\
\hline
\end{tabular}

AJCC American Joint Committee on Cancer, $H R$ hazard ratio, $C I$ confidence interval, $M F$ mass forming, $I G$ intraductal growth, $P I$ periductal infiltrating

TABLE 6 AJCC 8th edition and morphological type

\begin{tabular}{lccc}
\hline & $N=1083[n(\%)]$ & 5 -year OS $(\%)$ & $95 \%$ CI \\
\hline AJCC 8th edition T categories & $749(69.2)$ & 41.6 & $<0.001$ \\
T1a-T1b-T2 and MF/IG & $104(9.6)$ & 32.0 & $36.9-46.3$ \\
T1a-T1b-T2 and PI/MF + PI & $192(17.7)$ & 42.5 & $21.6-42.9$ \\
T3-T4 and MF/IG & $38(3.5)$ & 5.6 & $34.1-50.7$ \\
T3-T4 and PI/MF + PI & $N=1083[n(\%)]$ & HR & $0.5-20.6$ \\
\hline & & & CI Value \\
\hline AJCC 8th edition T categories & $749(69.2)$ & - & - \\
T1a-T1b-T2 and MF/IG & $104(9.6)$ & 1.47 & $1.11-1.94$ \\
T1a-T1b-T2 and PI/MF + PI & $192(17.7)$ & 1.01 & $0.79-1.28$ \\
T3-T4 and MF/IG & $38(3.5)$ & 3.59 & $2.46-5.23$
\end{tabular}

AJCC American Joint Committee on Cancer, $O S$ overall survival, $H R$ hazard ratio, $C I$ confidence interval, $M F$ mass forming, $I G$ intraductal growth, $P I$ periductal infiltrating

The current paper had several limitations that should be considered. The retrospective nature of the study may have resulted in selection bias; however, such confounding was unlikely to impact the evaluation of the prognostic effect of the morphological status. The multi-institutional nature of the study likely also caused some heterogeneity in ICC treatment approach. Finally, the pathological evaluation of ICC was conducted separately in each center, resulting in some heterogeneity in the interpretation of the pathological ICC characteristics. However, including multiple tertiary referral hepato-pancreatico-biliary (HPB) centers allowed for a large sample size and a more 'real-world' cohort.

\section{CONCLUSION}

Among patients undergoing curative-intent resection of ICC, morphologic subtype was associated with long-term outcome. In particular, patients with PI or MF + PI ICC had an approximately $45 \%$ increased risk of death compared with patients who had an MF or IG ICC. Interestingly, T3-T4 MF/IG patients had a similar risk of death as T1a-T1b-T2 MF/IG patients. Collectively, these data suggest that further refinements of staging, such as including tumor morphology, may be needed to better define the prognosis of patients with ICC.

DISCLOSURE The authors have no personal conflicts of interest to declare.

\section{REFERENCES}

1. Liver Cancer Study Group of Japan. Intrahepatic cholangiocarcinoma macroscopic typing. In: Okamoto E (ed). Classification of primary liver cancer. Tokyo, Japan, Kanehara; 1997. pp 6-7.

2. Dodson RM, Weiss MJ, Cosgrove D, et al. Intrahepatic cholangiocarcinoma: management options and emerging therapies. 
Journal of the American College of Surgeons. 2013;217(4): 736-50.

3. Amini N, Ejaz A, Spolverato G, Kim Y, Herman JM, Pawlik TM. Temporal trends in liver-directed therapy of patients with intrahepatic cholangiocarcinoma in the United States: a populationbased analysis. Journal of Surgical Oncology. 2014;110(2): 163-70.

4. de Jong MC, Nathan H, Sotiropoulos GC, et al. Intrahepatic cholangiocarcinoma: an international multi-institutional analysis of prognostic factors and lymph node assessment. Journal of Clinical Oncology. 2011;29(23):3140-45.

5. Hyder O, Hatzaras I, Sotiropoulos GC, et al. Recurrence after operative management of intrahepatic cholangiocarcinoma. Surgery. 2013;153(6):811-18.

6. Mavros MN, Economopoulos KP, Alexiou VG, Pawlik TM. Treatment and prognosis for patients with intrahepatic cholangiocarcinoma: systematic review and meta-analysis. JAMA Surgery. 2014;149(6):565-74.

7. Nathan H, Aloia TA, Vauthey JN, et al. A proposed staging system for intrahepatic cholangiocarcinoma. Annals of Surgical Oncology. 2009;16(1):14-22.

8. Nathan H, Pawlik TM. Staging of intrahepatic cholangiocarcinoma. Current Opinion in Gastroenterology. 2010;26(3):269-73.

9. Spolverato G, Ejaz A, Kim Y, et al. Tumor size predicts vascular invasion and histologic grade among patients undergoing resection of intrahepatic cholangiocarcinoma. Journal of Gastrointestinal Surgery. 2014;18(7):1284-91.

10. Spolverato G, Kim Y, Ejaz A, et al. Conditional probability of long-term survival after liver resection for intrahepatic cholangiocarcinoma: a multi-institutional analysis of 535 patients. JAMA Surgery. 2015;150(6):538-45.

11. Spolverato G, Vitale A, Cucchetti A, et al. Can hepatic resection provide a long-term cure for patients with intrahepatic cholangiocarcinoma? Cancer. 2015;121(22):3998-4006.

12. de Jong MC, Pulitano C, Ribero D, et al. Rates and patterns of recurrence following curative intent surgery for colorectal liver metastasis: an international multi-institutional analysis of 1669 patients. Annals of Surgery. 2009;250(3):44-48.

13. Spolverato G, Yakoob MY, Kim Y, et al. The impact of surgical margin status on long-term outcome after resection for intrahepatic cholangiocarcinoma. Annals of Surgical Oncology. 2015;22(12):4020-28.

14. Spolverato G, Kim Y, Alexandrescu S, et al. Is hepatic resection for large or multifocal intrahepatic cholangiocarcinoma justified? Results from a multi-institutional collaboration. Annals of Surgical Oncology. 2015;22(7):2218-25.

15. DeOliveira ML, Cunningham SC, Cameron JL, et al. Cholangiocarcinoma: thirty-one-year experience with 564 patients at a single institution. Annals of Surgery. 2007;245(5):755-62.

16. Endo I, Gonen M, Yopp AC, et al. Intrahepatic cholangiocarcinoma: rising frequency, improved survival, and determinants of outcome after resection. Annals of Surgery. 2008;248(1):84-96.
17. Nakagohri T, Kinoshita T, Konishi M, Takahashi S, Gotohda N. Surgical outcome and prognostic factors in intrahepatic cholangiocarcinoma. World Journal of Surgery. 2008;32(12):2675-80.

18. Bagante F, Gani F, Spolverato G, et al. Intrahepatic cholangiocarcinoma: prognosis of patients who did not undergo lymphadenectomy. Journal of the American College of Surgeons. 2015;221(6):1031-40.

19. Hatzaras I, Schmidt C, Muscarella P, Melvin WS, Ellison EC, Bloomston M. Elevated CA 19-9 portends poor prognosis in patients undergoing resection of biliary malignancies. $H P B$ (Oxford). 2010;12(2):134-38.

20. Yamasaki S. Intrahepatic cholangiocarcinoma: macroscopic type and stage classification. Journal of Hepato-Biliary-Pancreatic Surgery. 2003;10(4):288-91.

21. Shimada K, Sano T, Sakamoto Y, Esaki M, Kosuge T, Ojima H. Surgical outcomes of the mass-forming plus periductal infiltrating types of intrahepatic cholangiocarcinoma: a comparative study with the typical mass-forming type of intrahepatic cholangiocarcinoma. World Journal of Surgery. 2007;31(10):2016-22.

22. Ohtsuka M, Ito H, Kimura F, et al. Results of surgical treatment for intrahepatic cholangiocarcinoma and clinicopathological factors influencing survival. The British Journal of Surgery. 2002;89(12):1525-31.

23. Yamamoto M, Takasaki K, Yoshikawa T, Ueno K, Nakano M. Does gross appearance indicate prognosis in intrahepatic cholangiocarcinoma? Journal of Surgical Oncology. 1998;69(3): 162-67.

24. Amin MB EiC, American Joint Committee on Cancer. Springer, New York; 2017.

25. Austin PC, Grootendorst P, Anderson GM. A comparison of the ability of different propensity score models to balance measured variables between treated and untreated subjects: a Monte Carlo study. Statistics in Medicine. 2007;26(4):734-53.

26. Hyder O, Marques H, Pulitano C, et al. A nomogram to predict long-term survival after resection for intrahepatic cholangiocarcinoma: an Eastern and Western experience. JAMA Surgery. 2014;149(5):432-38.

27. Guglielmi A, Ruzzenente A, Campagnaro T, et al. Patterns and prognostic significance of lymph node dissection for surgical treatment of perihilar and intrahepatic cholangiocarcinoma. Journal of Gastrointestinal Surgery. 2013;17(11):1917-28.

28. Guglielmi A, Ruzzenente A, Campagnaro T, et al. Intrahepatic cholangiocarcinoma: prognostic factors after surgical resection. World Journal of Surgery. 2009;33(6):1247-54.

29. Spolverato G, Bagante F, Weiss, M, Alexandrescu S, Marques HP, Aldrighetti L, et al. Comparative performances of the 7th and the 8th editions of the American Joint Committee on Cancer staging systems for intrahepatic cholangiocarcinoma. Journal of Surgical Oncology. 